Annual percentage changes of total BMD $\left(\mathrm{g} / \mathrm{cm}^{2}\right)$ in the PF and LS were computed (annual $\triangle B M D$ ). The relationship between annual $\triangle B M D$ and potential predictors was assessed by independent-samples t-test or Pearson correlations, according to the categorical or continuous nature of the independent variable. The baseline independent variables included were: age (years); gender; body mass index $\left(\mathrm{kg} / \mathrm{m}^{2}\right)$; smoking, alcohol intake, rheumatoid arthritis, glucocorticoid treatment and secondary osteoporosis according to FRAX algorithm definitions. The follow-up reported calcium/vitamin D supplementation and treatment with bisphosphonates, for at least 1 year (yes/no), were also considered as independent variables. We used linear regression models (enter method) to estimate the possible association between socio-demographic/clinical variables and the BMD changes of PF and LS. The predictor variables included in the model were selected a priori based on variables that were associated with annual $\triangle B M D$ on univariate analysis. Results were considered significant when $p<0.05$.

Results: This analysis included data from 636 individuals (mean ( $\pm S D$ ) age of 53 \pm 8 years, $76 \%$ female). The mean ( $\pm S D$ ) follow-up period was $13.6 \pm 1.1$ years. Baseline mean $( \pm S D)$ BMD of the PF and LS were $0.77 \pm 0.004$ and $0.94 \pm 0.132$, respectively. The mean $( \pm S D)$ percentage of annual bone loss was: $-0.19 \pm 1.02$ for PF and $-0.06 \pm 0.84$ for LS. On univariate analysis, $\triangle B M D$ at both the PF and LS was significantly associated with gender $(p<0.001)$. Annual $\triangle B M D$ was also associated with vitamin $D$ supplements $(p=0.014)$ at $P F$, and with calcium supplements $(p=0.002)$ and bisphosphonate treatment $(p=0.027)$ at LS. On multiple linear regression analysis, female gender persisted as a predictor of faster BMD loss in both PF $(p \leq 0.001)$ and LS $(p=0.006)$. While bisphosphonate treatment didn't predict the annual bone loss, vitamin $D$ and calcium supplementation persisted as predictors of lower annual $\triangle B M D$ at $P F(p=0.034)$ and $L S(p=0.033)$, respectively. Conclusions: Our results confirm that the rate of BMD loss at PF and LS over 14 years is significantly associated with gender. Vitamin $D$ and calcium supplementation appeared to have a role in the prevention of bone loss in this general population cohort.

Disclosure of Interest: None declared

DOI: 10.1136/annrheumdis-2018-eular.5293

\section{THU0492 PRELIMINARY STUDY OF THE BONE MASS IN LUNG TRANSPLANTED PATIENTS IN TREATMENT WITH DENOSUMAB}

M. Barceló-Bru, B. Rodríguez-Diez. Rheumatology Unit, Hospital Universitari Vall d'Hebron, Barcelona, Spain

Background: Osteoporosis(OP) is a known complication before and after a lung transplant. In addition to altering bone mass, treatment with glucocorticoids alters the bone microarchitecture, conditioning an increased risk of fracture in these patients. Denosumab(Dmab) is a monoclonal antibody approved for the treatment of postmenopausal OP and its use for corticoid OP is pending approval.

Objectives: To describe the subcohort of lung transplant patients who have been treated with Dmab. To study the evolution of bone mass in patients with lung transplantation, with OP induced by glucocorticoids, treated with Dmab.

Methods: We included 19 lung transplant patients between 1995 and 2017 controlled in rheumatology for OP and in treatment with Dmab. Of these 19 patients, the evolution of bone mass of the 9 patients who have completed a minimum of 12 months of treatment is shown. Bone densitometry(DXA) was performed on a General Electric Healthcare Lunar Prodigy Advance 15 version densitometer, before and after treatment with Dmab. Demographic data of the patients were collected, the diagnosis of the disease that motivated the transplant, the glucocorticoid doses before and after the transplant, as well as the immunosuppressive treatment. In the 9 patients with DXA follow-up, the 3D-SHAPER software was applied in the DXA before and after the transplant.

Results: We included 19 patients(10 women) with a mean age of 58.6 years \pm 11.4 . The diagnosis of the disease that led to the transplant was in 4 COPD patients, 6 patients with pulmonary fibrosis or diffuse interstitial disease, 2 with bronchiectasis, 2 patients with cystic fibrosis, 1 with histiocytosis X, 2 with lymphangioleiomyomatosis, 1 with bronchiolitis obliterans and 1 patient with pulmonary hypertension. Before transplantation, 8 patients $(42.1 \%)$ had required high doses of glucocorticoids. The prevalence of OP prior to start Dmab treatment in the 19 lung transplant patients was $94.7 \%$. The means of BMD in $\mathrm{g} / \mathrm{cm}^{2}$ and Tscore before treatment with Dmab and the results of the 3D-SHAPER are shown in table 1.

\begin{tabular}{lcc}
\hline & $\mathrm{DMO} \mathrm{g} / \mathrm{cm}^{2}$ & T-score \\
\hline Lumbar spine & $0,909 \pm 0,13$ & $-2,40 \pm 1,08$ \\
Femoral Neck & $0,708 \pm 0,94$ & $-2,50 \pm 0,77$ \\
Total Hip & $0,702 \pm 0,86$ & $-2,73 \pm 0,59$ \\
Cortical Density $\left(\mathrm{g} / \mathrm{cm}^{2}\right)$ & $109,3 \pm 7,7$ & $-2,9 \pm 0.3$ \\
Trabecular vBMD $\left(\mathrm{g} / \mathrm{cm}^{3}\right)$ & $85,7 \pm 21,4$ & $-2,9 \pm 0.8$ \\
Integral vBMD $\left(\mathrm{g} / \mathrm{cm}^{3}\right)$ & $198,7 \pm 19$ & $-3,3 \pm 0,5$ \\
\hline
\end{tabular}

The mean time in which the DXA was performed prior to the treatment with Dmab with respect to the transplant date was $4.31 \pm 5.83$ years. The mean time in which the DXA was performed after the start of Dmab was $19 \pm 4.17$ months. The results of the evolutionary BMD, the 3D-SHAPER study and its percentage of change are shown in table 2 . Of these 9 patients, $88.9 \%$ had received osteoactive treatment before Dmab, 5 with oral bisphosphonates and 3 with endovenous bisphosphonates. The mean time of treatment with bisphosphonates until the onset of Dmab was 5.4 years. There were no significant differences in BMD evolution between patients treated for OP prior to Dmab and those not treated, nor for 3D-SHAPER.

\begin{tabular}{|c|c|c|}
\hline & \multicolumn{2}{|c|}{$\mathrm{Tx}(9)$} \\
\hline OP pre-Dmab & \multicolumn{2}{|c|}{$8(88,9 \%)$} \\
\hline OP post-Dmab & \multicolumn{2}{|c|}{$8(88,9 \%)$} \\
\hline \begin{tabular}{ll}
\multicolumn{2}{l}{ DMO } \\
$-\quad$ post-Dmab (mean) \\
$-\quad$ LF \\
$-\quad$ CF \\
$-\quad$ FT
\end{tabular} & $\begin{array}{c}\mathrm{g} / \mathrm{cm}^{2} \\
0,949 \pm 0,13 \\
0,722 \pm 0,12 \\
0,696 \pm 0,06\end{array}$ & $\begin{array}{c}\text { T-score } \\
-2,05 \pm 1,07 \\
-1,86 \pm 2,22 \\
-2,76 \pm 0,49\end{array}$ \\
\hline $\begin{array}{lc}\% & \text { change DMO } \\
- & \text { L1-L4 } \\
- & \text { CF } \\
- & \text { FT } \\
\end{array}$ & \multicolumn{2}{|c|}{$\begin{array}{l}8,42 \pm 6,64^{*} \\
2,21 \pm 6,55^{*} \\
2,80 \pm 3,56^{*}\end{array}$} \\
\hline High GC dose pre-Transplant & \multicolumn{2}{|c|}{$2(22,2 \%)$} \\
\hline $\begin{array}{ll}\text { 3D-SHAPER (mean): } \\
-\quad \text { Cortical Density }\left(\mathrm{g} / \mathrm{cm}^{2}\right) \\
-\quad \text { Trabecular vBMD }\left(\mathrm{g} / \mathrm{cm}^{3}\right) \\
- & \text { Integral vBMD }\left(\mathrm{g} / \mathrm{cm}^{3}\right) \\
\end{array}$ & \multicolumn{2}{|c|}{$\begin{array}{c}113,7 \pm 10 \\
88,5 \pm 23.7 \\
203,7 \pm 24,4 \\
\end{array}$} \\
\hline $\begin{array}{ll}\% \text { change 3D-SHAPER: } \\
-\quad & \text { Cortical Density } \\
- & \text { Trabecular VBMD } \\
- & \text { Integral vBMD } \\
\end{array}$ & \multicolumn{2}{|c|}{$\begin{array}{c}4,13 \pm 4,5 \\
3,4 \pm 10,9 \\
2,6 \pm 7,7\end{array}$} \\
\hline
\end{tabular}

Conclusions: All patients who started treatment with Dmab had a diagnosis of OP except one patient with osteopenia. The mean increase in BMD in patients treated with Dmab was significant in all the regions assessed, being higher in the lumbar spine. There was an increase in cortical density, trabecular volumetric BMD and integral volumetric BMD after Dmab treatment, although this increase was not significant for any of the three parameters.

Disclosure of Interest: None declared

DOI: 10.1136/annrheumdis-2018-eular.4717

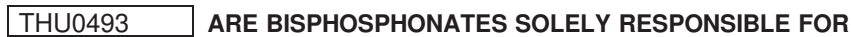 ATYPICAL FEMORAL FRACTURES? A CASE SERIES}

M.S. Jamal, C. Cockill, S. knights. Rheumatology, YDH, Yeovil, UK

Background: Atypical femoral fracture (AFF) is an uncommon but important type of fracture. It is concerning because of the associated morbidity, bilateral nature of the condition and delay in healing which has significant implications on the health of the patients.

Objectives: To identify other risk factors contributing to development of atypical femoral fractures in our cohort of patients presenting to the fracture liaison service.

Methods: We identified a case series of 18 cases were retrospectively from June 2006 to January 2018 through the cohort of patients presenting to the fracture liaison service at Yeovil District Hospital NHS foundation trust, with features suggestive of Atypical femoral fractures. Individual patient cases were evaluated and notes and images reviewed. Atypical femoral fractures were diagnosed according to the 2013 American society of mineral and bone research (ASMBR) criteria. Demographic and co morbidity status was investigated from case notes. Blood results and dual enerty $x$-ray absorbtiometry (DEXA) images were accessed through electronic patient records. The results were compiled with Microsoft excel.

Results: There were total 22 patient episodes, of which 4 patients had bilateral symptoms. Female to male ratio was 21:1. Median age of presentation was 71 The incidence of AFF was more in shaft fractures. $54 \%$ cases had prodromal symptoms lasting from 1-3 months. 9 cases had radiographic evidence of stress fractures of whom 6 were not identified until they fractured. $86 \%$ cases had either concurrent or previous bisphosphonate therapy. Duration of Bisphosphonate therapy was variable from 12 months to 30 years. 54\% were taking Proton pump inhibitors and $41 \%$ were on long term glucocorticoids. $9 \%$ were active smokers. $50 \%$ episodes were in patients having two or more risk factors. 3 patients had no risk factors identified. 